\title{
A Case of Overt GI Bleeding from a Obscured Source in a Cirrhotic Patient
}

Deepankar Kumar Basak ${ }^{1}$, Md. Samsul Arfin², Mohammed Motahar Hossain ${ }^{3}$

\begin{abstract}
:
While esophago-gastric varices are common manifestations of portal hypertension, variceal bleeding from the jejunum is a rare complication of liver cirrhosis. In addition, ectopic variceal bleeding occurs in the duodenum and at sites of previous bowel surgery in most cases, including of stomas. We report a case of obscure overt gastrointestinal bleeding from jejunal varices in a 70-year-old woman who had not previously undergone abdominal surgery, who had not diagnosed liver cirrhosis before. Emergency endoscopy revealed no esophageal varices but multiple nodular lesions with ulcerated tips with active oozing of blood and no bleeding focus was found at colonoscopy. She continued to produce recurrent melaena with hematochezia and received several units of packed red blood cells. CT angiography revealed the presence of jejunal varices, but no active bleeding was found. USG\&CT scan of abdomen reveals coarse liver with splenomegally with mild pelvic collection. This is a case of variceal bleeding from the jejunum in a liver cirrhosis patient without a prior history of abdominal surgery.
\end{abstract}

Keywords: Jejunal varices; Liver cirrhosis; Portal hypertension

\section{INTRODUCTION}

Varices commonly occur in the gastro-esophageal region and bleeding from gastro-esophageal varies is one of the most serious complication of portal hypertension. However, ectopic varies can arise anywhere in the abdomen except in the cardio-esophageal region and can represent an unusual cause of hemorrhage, accounting for up to $5 \%$ of all variceal bleeding. ${ }^{1}$ Although ectopic varices bleed less commonly than gastro-esophageal varices, they can be far more difficult to diagnose and treat. Ectopic varices can occur at several sites; bleeding ectopic varies are most commonly found in the duodenum and at sites of previous bowel surgery including stomas. ${ }^{1,2}$ However, varicel bleeding from the jejunum in a cirrhotic patient without a prior history of abdominal surgery is rare. We report a case of intrahepatic portal hypertension-related variceal bleeding from the jejunum with obscure overt gastrointestinal bleeding who has not previously undergone abdominal surgery and review the literature pertaining to this condition.

\section{Dr. Deepankar Kumar Basak, MBBS, FCPS Specialist \\ 2. Prof. Dr. Md. Samsul Arfin, MBBS, FCPS Consultant \\ 3. Dr. Mohammed Motahar Hossain, MBBS, FCPS, MD Consultant}

All authors work in Department of Hepatology \& Gastroenterology Square Hospitals Ltd, Dhaka, Bangladesh.

\section{Corresponding Author:}

Dr. Deepankar Kumar Basak, MBBS, FCPS Specialist

Department of Hepatology \& Gastroenterology

Square Hospitals Ltd,

18F B U Kazi Nuruzzaman Sarak

Dhaka, Bangladesh.

E mail: deepankarbasak@gmail.com

\section{CASE REPORT}

A 70-year-old diabetic \& hypertensive patient was admitted to SHL through ER with a history of passage of black tarry stool and occasional fresh per rectal bleeding for last 15 days. She gave no history of bleeding from any site of the body, abdominal pain, fever, jaundice, weight loss, altered bowel habit and also taking any NSAIDs, anti-coagulant, aspirin or steroid. With above complaints was $1^{\text {st }}$ admitted to a local hospital and underwent endoscopy \& colonoscopy which revealed no abnormality but the symptom persist and was shifted to SHL gastro-department for further evaluation. In admission she was pale, not icteric well oriented with blood pressure $110 / 70 \mathrm{mmHg}$, pulse- $80 / \mathrm{min}$. On investigation Hb-6.6g/dl,Hct-19.7\%,TC-6.11K/uL,PT-192K/uL,Bilirubin$1.4 \mathrm{mg} / \mathrm{dl}, \mathrm{ALT}-50 \mathrm{u} / 1$, AST-70u/1,INR-1.10sec,aPTT-24.1,A:G ratio altered and all viral markers was negative. After volume replacement \& correction of hemoglobin, she again underwent upper GI endoscopy and found no esophageal varices (Fig1) but multiple nodular lesions with ulcerated tips with evidence of active oozing of blood at the proximal

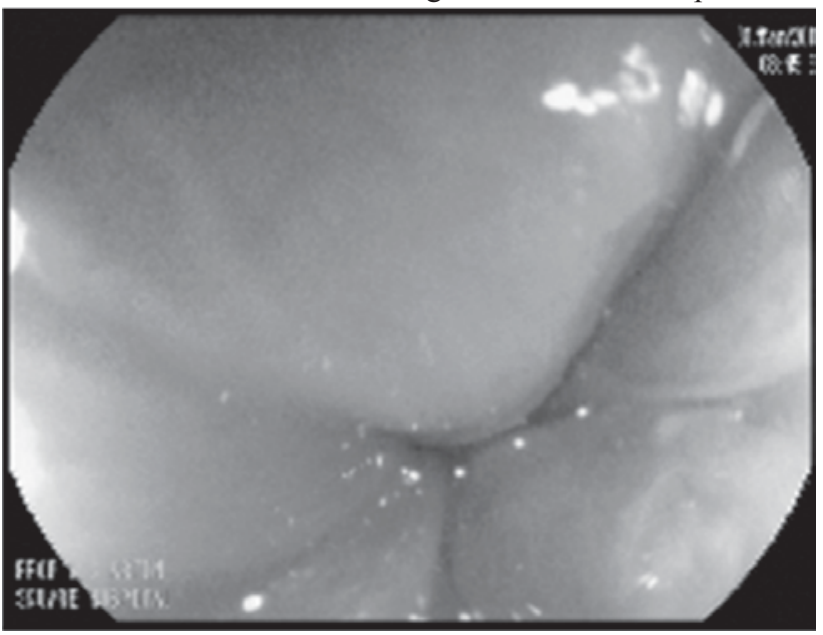

Fig1-No esophageal varices 
jejunum (Fig2,3). We performed injection sclerotherapy with 9 cc of ethanolamine oleate and the bleeding stopped. Colonoscopy revealed rectal polyp with hemorrhoids. Melaena again started after 2 days. We want to do an again upper GI endoscopy but they refused to do that.

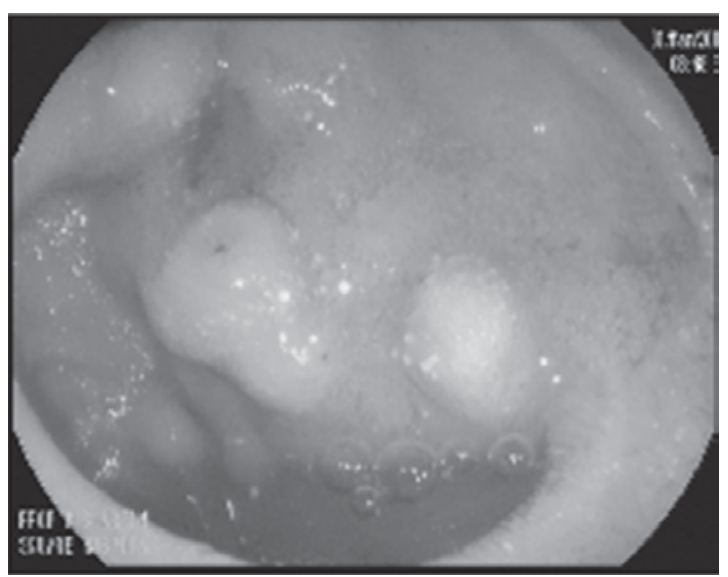

In the mean time we do CT scan \&USG of abdomen which reveals liver is irregular, spleen is enlarged \& mild pelvic collection (Fig4).After that patient undergo CT angiography and revealed a tortuous venous channel is detected at the Right side of the abdomen communicating between the

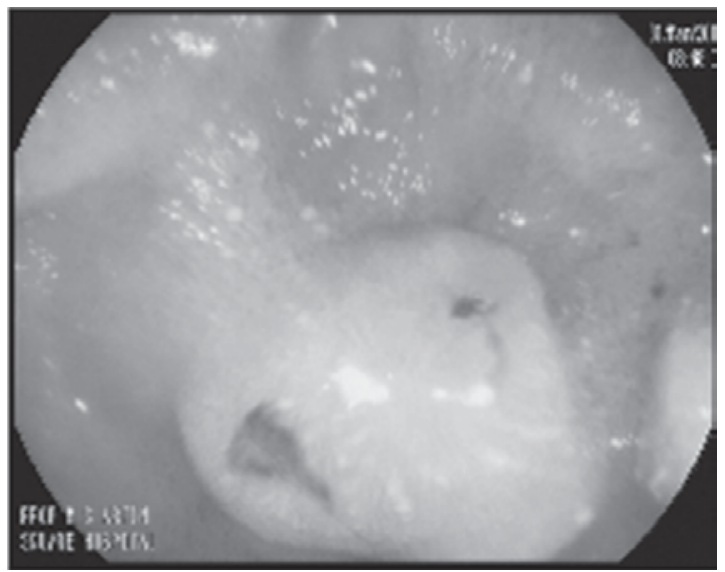

Fig 2-multipple nodular lesions in the proximal jejunum
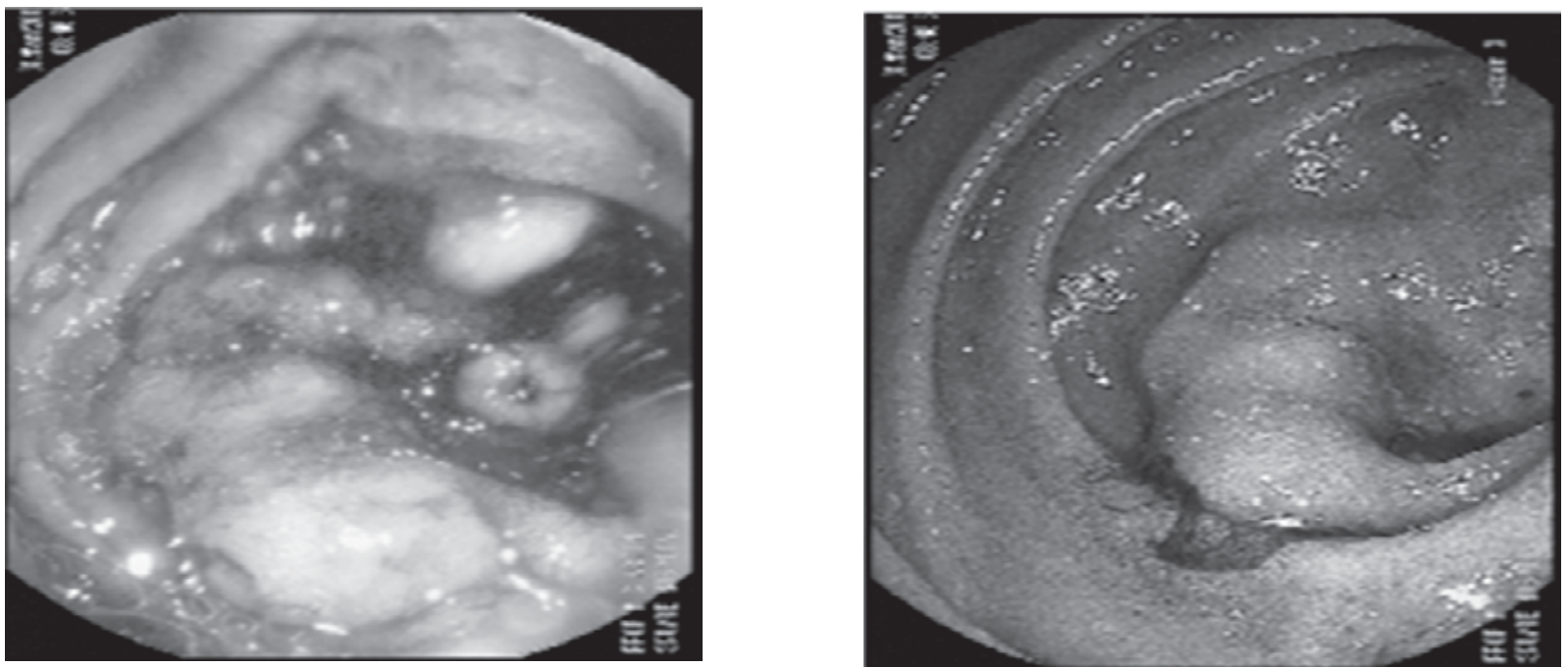

Fig 3-active bleeding from jejuna varices

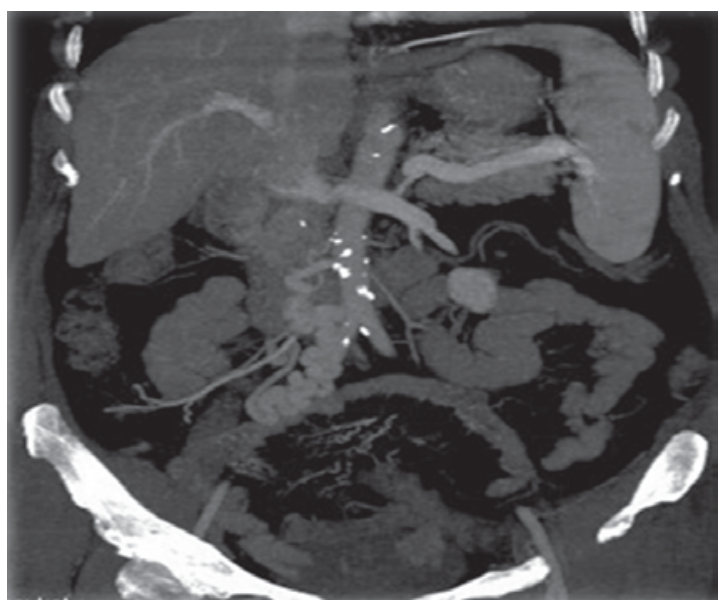

Fig 4-CT abdomen showing irregular liver with splenomegally with mild pelvic collection superior mesenteric vein and IVC with ramification within the wall of jejunum suggesting prominent Porto-systemic anastomosis traversing the jejuna wall (Fig5). We do fibro scan revealed score 39.Biopsy from the lesion revealed chronic jejunitis. So we finally reached to a diagnosis that melaena due to ectopic varices in the jejunum due to cryptogenic cirrhosis. Melaena was still continuing. We started beta-blocker and stlilamin infusion for bleeding. Melaena was stopped but she needs further intervention for definite treatment.

\section{DISCUSSION}

Obscure GI bleeding could be categorized as obscure overt and obscure occult bleeding on the presence or absence of clinically evident bleeding. ${ }^{3}$ It has been estimated that approximately $5 \%$ of GI bleeding occurs in the small intestine ${ }^{4}$ Lesions caused by portal hypertension are also 

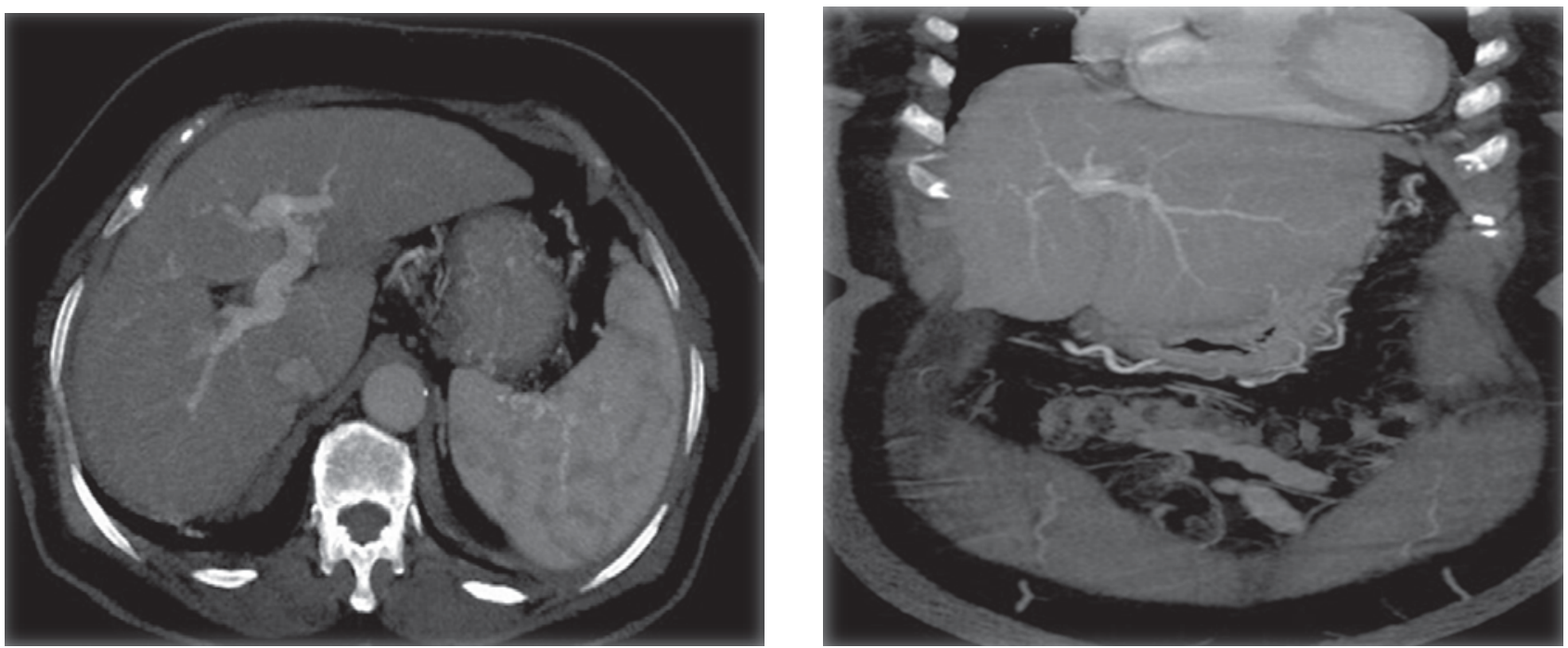

Fig 5-CT angiogram of abdomen showing Jejunal varices

important causes of obscure bleeding. Varices, either in the small bowel or in other unusual locations, should be considered in all obscure bleeders who have liver disease and/or portal hypertension ${ }^{4}$ Ectopic varices are defined as large port systemic venous collaterals occurring anywhere in the abdomen except in the cardio-esophageal region. In patients with portal hypertension caused by an obstruction of the portal or splenic veins, duodenal varices are more prevalent than in patients with intrahepatic portal hypertension. ${ }^{1}$ A history of abdominal surgery appears to predispose the development of ectopic varices in adhesions ${ }^{2}$ A triad of portal hypertension, history of abdominal surgery and hematochezia without hematemesis characterizes small intestinal varices. ${ }^{2}$ Duodenal varices occur in about $0.4 \%$ of all patients with portal hypertension and account for one-third of bleeding episodes from ectopic varices. Variceal bleeding from the jejunum is more rare than variceal bleeding from the duodenum because of inaccessibility and anatomic difference. ${ }^{5}$ Recently, smallbowel varices secondary to portal hypertension, diagnosed by capsule endoscopy, are reported and small bowel varices and mucosal changes are considered the definite or probable source of bleeding. ${ }^{6}$ Investigations of the small intestine, including radiographic contrast studies, nuclear scans, angiography, capsule endoscopy and push enteroscopy have been used to diagnose jejunal varices. ${ }^{7}$ In this case, CT angiography was negative and a capsule endoscopy was performed as the next step. Capsule endoscopy, which is relatively non-invasive, was a suitable investigation for this patient.

Capsule endoscopy is being used increasingly to investigate GI bleeding of obscure origin and disorders of the small bowel. Tang SJ et al. found small bowel varices in four out of 46 patients $(8.7 \%)$ who underwent capsule endoscopy for obscure GI bleeding. ${ }^{8}$ They reported that capsule endoscopy is invaluable and highly sensitive for the detection of small-bowel variceal bleeding. Triester SL et al. reported that capsule endoscopy was shown to be superior to push enteroscopy in a meta-analysis, although it has limitations in its inability to therapy. ${ }^{9}$

The initial management of bleeding from jejunal varices involves hemodynamic stabilization with correction of anemia and coagulopathy. The patient was treated with IV stilamin, which has been beneficial in the management of bleeding esophageal varices. The role of stilamin in the control of bleeding from jejunal varices has not been estimated, but the use of stilamin to reduce splanchnic blood flow and variceal pressure may be beneficial in patients with intrahepatic portal hypertension-related variceal bleeding. ${ }^{1}$

Outside the reach of standard endoscopy, available therapy for jejunal variceal bleeding is limited to surgery, transjugular intrahepatic porto-systemic shunt (TIPS), enteroscopy and percutaneous emolization. Surgery has been the main treatment option of bleeding jejunal varices. ${ }^{10-12}$ the embolization of coil can be attempted in this case because she had coagulopathy with high risk associated with surgery. Radiological techniques with embolization have been used successfully in the active bleeding of jejunal varices. ${ }^{13}$ There is a case report of enteroscopic sclerotherapy being successfully carried out using cyanoacrylate to treat hemorrhage from jejuna and gallbladder varices. ${ }^{14}$ The selection of possible therapeutic options for cirrhotic patients was made considering the risks additive benefits in the situation and the health of the patients In conclusion, bleeding from ectopic varices must be considered for patients with portal hypertension and obscure overt GI bleeding, and we described a case of variceal bleeding from the jejunum in a patient with liver cirrhosis who had not previously undergone abdominal surgery. Combination of CT angiography and capsule endoscopy could lead towards diagnosis.

\section{REFERENCES}

1. Norton ID, Andrews JC, Kamath PS. Management of ectopic varices. Hepatology 1998; 28:1154-1158. 
Bangladesh Crit Care J September 2014; 2 (2): 81-84

2. Sharma D, Misra SP. Ectopic varices in portal hypertension. Indian J Surg 2005; 67:246-252.

3. Raju GS, Gerson L, Das A, Lewis B; American Gastroenterological Association. American Gastroenterological Association (AGA) Institute technical review on obscure gastrointestinal bleeding. Gastroenterology 2007; 133:1697-1717.

4. Rockey DC. Occult and obscure gastrointestinal bleeding: causes and clinical management. Nat Rev Gastroenterol Hepatol 2010; $7: 265-279$

5. Almadi MA, Almessabi A, Wong P, Ghali PM, Barkun A. Ectopic varices. Gastrointest Endosc 2011; 74:380-388.

6. De Palma GD, Rega M, Masone S, Persico F, Siciliano S, Patrone F, et al. Mucosal abnormalities of the small bowel in patients with cirrhosis and portal hypertension: a capsule endoscopy study. Gastrointest Endosc 2005; 62:529-534.

7. Leighton JA, Goldstein J, Hirota W, Jacobson BC, Johanson JF, Mallery JS, et al. Obscure gastrointestinal bleeding. Gastrointest Endosc 2003; 58:650-655.

8. Tang SJ, Zanati S, Dubcenco E, Cirocco M, Christodoulou D, Kandel G, et al. Diagnosis of small-bowel varices by capsule endoscopy. Gastrointestinal Endosc 2004; 60:129-135.
9. Triester SL, Leighton JA, Leontiadis GI, Fleischer DE, Hara AK, Heigh RI, et al. A meta-analysis of the yield of capsule endoscopy compared to other diagnostic modalities in patients with obscure gastrointestinal bleeding. Am J Gastroenterology 2005; 100:2407-2418.

10. Joo YE, Kim HS, Choi SK, Rew JS, Kim HR, Kim SJ. Massive gastrointestinal bleeding from jejunal varices. J Gastroenterol 2000; $35: 775-778$

11. Deshpande A, Sampat P, Bhargavan R, Sharma M. Bleeding isolated jejunal varices without portal hypertension. ANZ J Surg 2008; 78:814-815.

12. Bykov S, Becker A, Koltun L, Yudko E, Garty I. Massive bleeding from jejunal varices in a patient with thalassemia major detected by TC-99m red blood cell scintigraphy. Clin Nucl Med 2005; 30:457459.

13. Lim LG, Lee YM, Tan L, Chang S, Lim SG. Percutaneous paraumbilical embolization as an unconventional and successful treatment for bleeding jejunal varices. World J Gastroenterol 2009; 15:3823-3826.

14. Getzlaff S, Benz CA, Schilling D, Riemann JF. Enteroscopic cyanoacrylate sclerotherapy of jejunal and gallbladder varices in a patient with portal hypertension. Endoscopy 2001; 33:462-464. 